\title{
Répartition et évolution des éléments minéraux au cours de l'affinage des fromages à pâte cuite type Beaufort
}

\author{
Y. LE GRAET*, G. BRULÉ, J.-L. MAUBOIS* et G. EEUVRARD** \\ * I.N.R.A., Laboratoire de Recherches de Technologie laitière \\ 65, rue de Saint-Brieuc, 35042 Rennes cedex, France \\ ** Coopérative laitière du Beaufortain, 73270 Beaufort-sur-Doron, France
}

\section{Résumé}

Au cours de l'affinage des fromages à pâte cuite de type Beaufort, le calcium, le magnésium, le zinc et le fer migrent de l'intérieur vers la morge. Les teneurs respectives en calcium et magnésium sont de $41,31 \mathrm{~g} / \mathrm{kg}$ et $1,17 \mathrm{~g} / \mathrm{kg}$ dans la morge, $8,07 \mathrm{~g} / \mathrm{kg}$ et $0,24 \mathrm{~g} / \mathrm{kg}$ dans le centre ; celles du fer et du zinc sont de $27 \mathrm{mg} / \mathrm{kg}$ et $170 \mathrm{mg} / \mathrm{kg}$ dans la morge, $2 \mathrm{mg}$ et $45 \mathrm{mg} / \mathrm{kg}$ dans le centre.

La présence d'importantes quantités de $\mathrm{CO}_{2}$ associées aux minéraux dans la morge permet de penser que le calcium et le magnésium se trouvent sous forme de carbonates insolubles dont la formation est favorisée par un $\mathrm{pH}$ de surface basique.

La morge est beaucoup plus riche en sodium, $9,85 \mathrm{~g} / \mathrm{kg}$, et en potassium, $2,78 \mathrm{~g} / \mathrm{kg}$, que le centre du fromage où les teneurs sont respectivement de $4,73 \mathrm{~g} / \mathrm{kg}$ et $0,92 \mathrm{~g} / \mathrm{kg}$.

Le cuivre migre peu puisque la teneur de la morge est de $16,25 \mathrm{mg} / \mathrm{kg}$ et celle du centre, $7,87 \mathrm{mg} / \mathrm{kg}$.

L'analyse des saumures utilisées pour les emmorgeages successifs montre qu'elles s'enrichissent en minéraux, essentiellement calcium, magnésium, zinc et fer au fur et à mesure de leur utilisation; le transfert minéral se fait donc de la pâte vers la saumure, mais en quantité négligeable par rapport à la teneur minérale des fromages.

L'étude de l'évolution du calcium et du phosphore au cours de l'affinage montre que, dès que le fromage atteint 15 jours d'âge, seul le premier migre de l'intérieur vers l'extérieur pour former un carbonate.

Mots clés : Affinage - Beaufort - Minéraux - Phosphore - Calcium - Magnésium Sodium - Potassium - Zinc - Fer - Cuivre.

\section{Summary}

Distribution and evolution of mineral substances during ripening of cooked hard cheese of the Beaufort type

During ripening of cooked hard cheese of the Beaufort type, calcium, magnesium, zinc and iron move from inside to bacterial smear ; calcium and magnesium amounts are respectively $41.31 \mathrm{~g} / \mathrm{kg}$ and $1.17 \mathrm{~g} / \mathrm{kg}$ in smear, $8.07 \mathrm{~g} / \mathrm{kg}$ and $0.24 \mathrm{~g} / \mathrm{kg}$ in the middle part. Iron and zinc amount are $27 \mathrm{mg}$ and $170 \mathrm{mg} / \mathrm{kg}$ in smear, $2 \mathrm{mg}$ and $45 \mathrm{mg} / \mathrm{kg}$ in the middle part. 
The migration of these elements results from the formation of insoluble carbonates or hydroxydes favoured by high $\mathrm{pH}$ and by surface treatment. The mineral content of the brine used for rubbing the cheese surface increases only slightly, especially in calcium, magnesium, zinc and iron.

Investigation on the evolution of calcium and phosphorus, during cheese ripening, shows the migration of calcium starts from the fifteenth day onwards.

Key words : Ripening - Hard cheese - Minerals - Phosphorus - Calcium - Magnesium Sodium - Potassium - Zinc - Iron - Copper.

\section{Introduction}

Le rôle de la fraction minérale sur l'aptitude des laits à coaguler et les caractéristiques théologiques du caillé et du fromage est reconnu de tous. La composition minérale d'un fromage dépend étroitement des différents paramètres technologiques mis en cuvre lors de sa fabrication; l'évolution des technologies, au cours de ces dernières décades, dans l'industrie fromagère en général, explique probablement, au moins partiellement, celle de la composition minérale que nous pouvons appréhender en comparant les données de GuitTonNeaU et CHEvalier (1934) et celles de Gueguen (1979). Nous savons en outre, depuis les travaux de Metche et Fanni (1978) et Le Graet et al. (1983), qu'au cours de l'affinage de fromages à pâte molle de type Camembert certains éléments migrent vers la surface, ce qui entraîne une très grande hétérogénéité dans la composition minérale de la pâte. Cette migration induite par les caractéristiques physico-chimiques de la croûte et notamment son $\mathrm{pH}$ est étroitement dépendante de l'atmosphère des caves d'affinage et des soins de surface.

Aucune étude, à l'exception de celles concernant les éléments sodium, calcium et phosphore (Koestler, 1943 ; Mattson, 1954 ; Resmini et al., 1974) n'ayant été réalisée sur la répartition des différents éléments de la fraction minérale dans un fromage à pâte cuite, nous avons entrepris, d'une part, d'établir la distribution des macroéléments $(\mathrm{P}, \mathrm{Ca}, \mathrm{Mg}, \mathrm{K}, \mathrm{Na})$ et des oligoéléments $(\mathrm{Zn}, \mathrm{Cu}$, Fe) dans des fromages de Beaufort affinés et, d'autre part, de suivre la migration des macroéléments au cours des premières semaines d'affinage. Ce type de fromage nous a paru particulièrement intéressant étant donné l'importance des soins de surface et le caractère ammoniacal marqué de l'atmosphère des caves d'affinage.

\section{Matériel et méthodes}

- Fromages. Les échantillons de fromage étaient fournis par la Coopérative du Beaufortain sous forme d'une demi-tranche radiale de fromage âgé de 8 mois pour l'étude de la répartition minérale, et de sondes prélevées à mi-rayon pour l'étude de la migration minérale.

- Découpe. Une tranche de $30 \mathrm{~mm}$ d'épaisseur, coupée de part et d'autre d'un diamètre du fromage et ensuite divisée en son centre dans le sens de la hauteur en deux moitiés égales dont l'une servait au prélèvement des échantillons. Cette demi-tranche était découpée, horizontalement en lamelles de $11 \mathrm{~mm}$ parallèles à la croûte, et verticalement à partir du talon, en 3 tranches successives de $10 \mathrm{~mm}$, 
puis 2 tranches de $20 \mathrm{~mm}, 1$ tranche de 40 et $60 \mathrm{~mm}$ et le reste $80 \mathrm{~mm}$ environ (fig. 1). Sur la couche externe de $11 \mathrm{~mm}$ étaient prélevés $3 \mathrm{~mm}$ représentant la croûte. Les résultats présentés ont été obtenus en effectuant le rapport :

$\Sigma$ (concentration de l'élément dans l'échantillon) (poids de l'échantillon)

$\Sigma$ (poids de chaque échantillon)

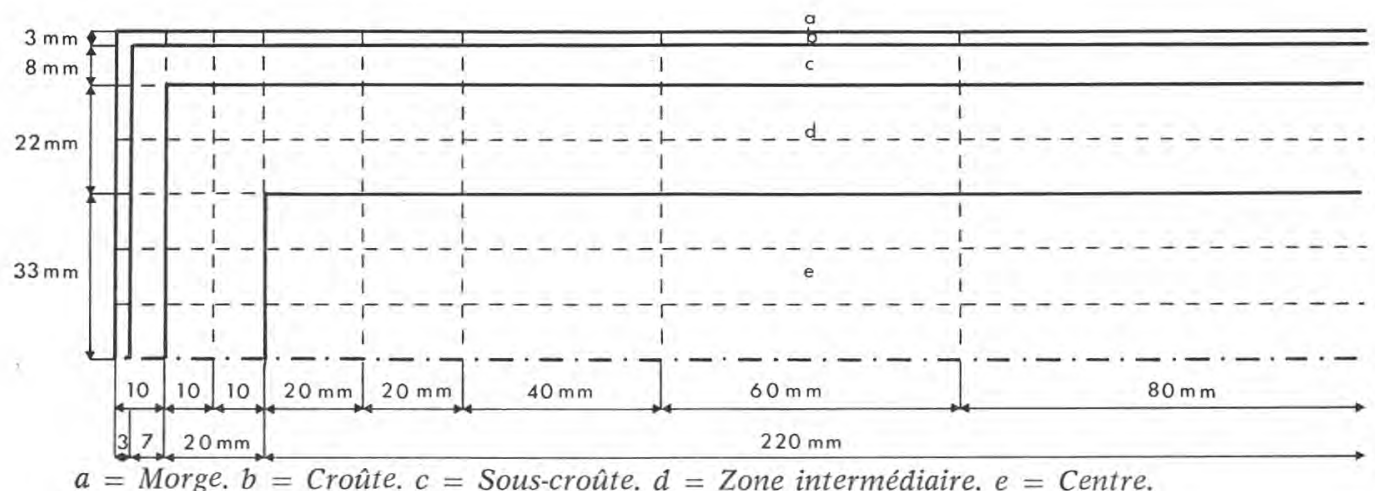

Fig. 1

Découpe du fromage - Cutting of cheese.

et exprimés en $\mathrm{g}$ ou $\mathrm{mg} / \mathrm{kg}$ pour chacune des zones : croûte (1b), partie sous croûte (1c), zone intermédiaire (1d), et le centre du fromage (1e).

La morge était obtenue en raclant la couche superficielle du fromage sur lequel était prélevé l'échantillon, mais sur une surface supérieure à celle du prélèvement de façon à obtenir une quantité suffisante.

Les sondes étaient découpées en 3 parties : la croûte, la partie sous-croûte et le centre (fig. 2) ; ces 3 dénominations ont une signification différente du cas précédent (notamment en ce qui concerne le centre).

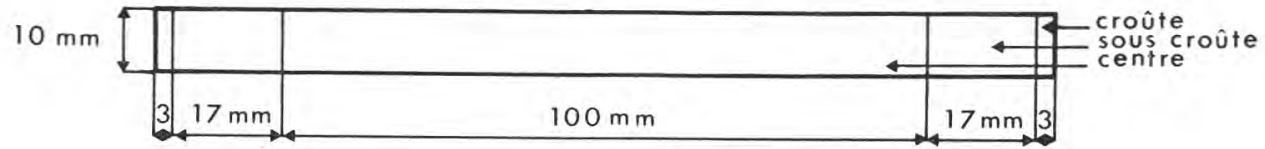

Fig. 2

Prélèvement pour l'étude de la migration minérale au cours de l'affinage.

Sample for study of mineral migration during ripening.

\section{Déterminations analytiques}

- Cendres. Elles sont obtenues par calcination d'environ $2 \mathrm{~g}$ de fromage ou de $0,5 \mathrm{~g}$ de croûte à $530^{\circ} \mathrm{C}$ pendant $3 \mathrm{~h}$. 
- Teneurs en minéraux. Les déterminations des teneurs en calcium, magnésium, sodium, potassium, fer, zinc et cuivre étaient réalisées par spectrophotométrie d'absorption atomique sur un appareil VARIAN AA 1275 à partir des cendres obtenues sur les fromages et reprises dans l'acide chlorhydrique $0,1 \mathrm{~N}$. A partir de cette solution les dilutions étaient réalisées en fonction de la teneur en chacun des éléments.

Le dosage du phosphore total était effectué à partir de $5 \mathrm{ml}$ de la solution précédente, selon la méthode préconisée par la Fédération Internationale de Laiterie (norme 33 ).

- Teneurs en matière sèche. Elle était déterminée par dessiccation à l'étuve à $102-105^{\circ} \mathrm{C}$ pendant $7 \mathrm{~h}$.

- Mesure du pH. Les déterminations de $\mathrm{pH}$ étaient effectuées avec un appareil PORTATEST 653 à 0,02 unité $\mathrm{pH}$ près.

\section{Résultats}

A. Répartition des éléments minéraux dans le fromage affiné

\section{Caractéristiques physico-chimiques du fromage}

Le fromage présente une très grande hétérogénéité quant à ses caractéristiques physico-chimiques comme le montre la figure 3 . Il existe un gradient
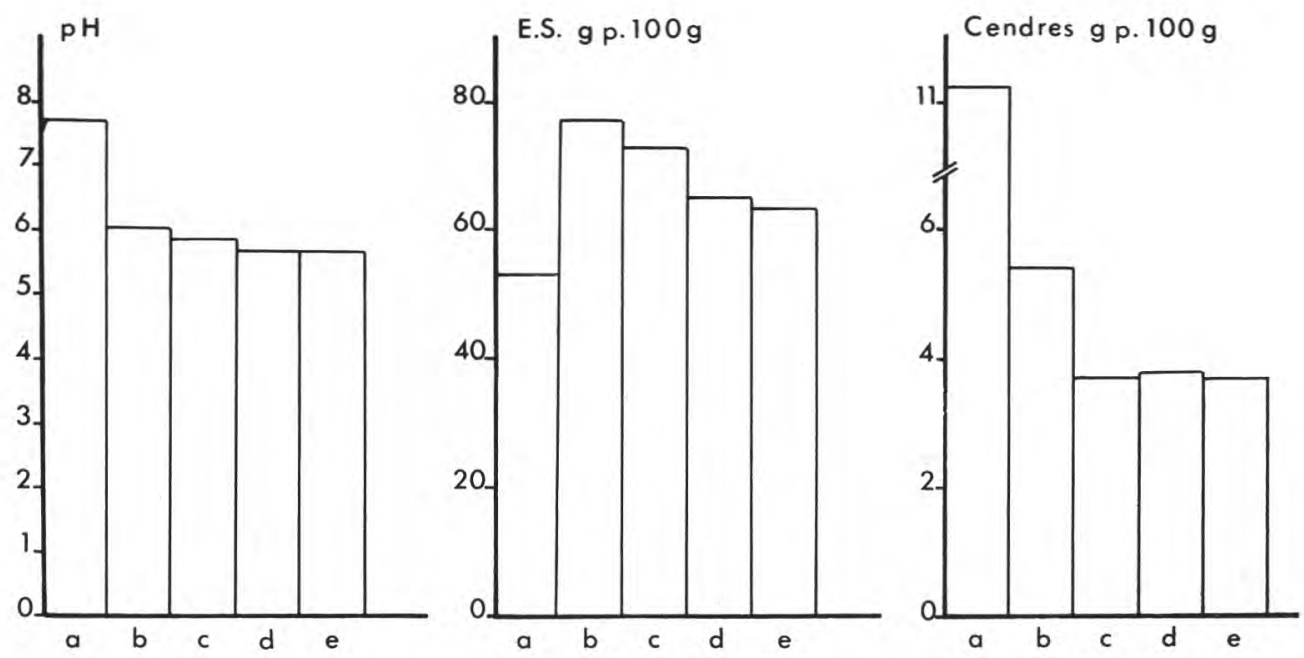

Fig. 3

$p H$, extrait sec et cendres d'un fromage affiné. $p H$, dry matter and ash in the ripened cheese.
$\mathrm{a}=$ Morge.
$\mathrm{b}=$ Croûte.
$\mathrm{d}=$ Zone intermédiaire .
$\mathrm{c}=$ Sous-croûte.
$\mathrm{e}=$ Centre . 

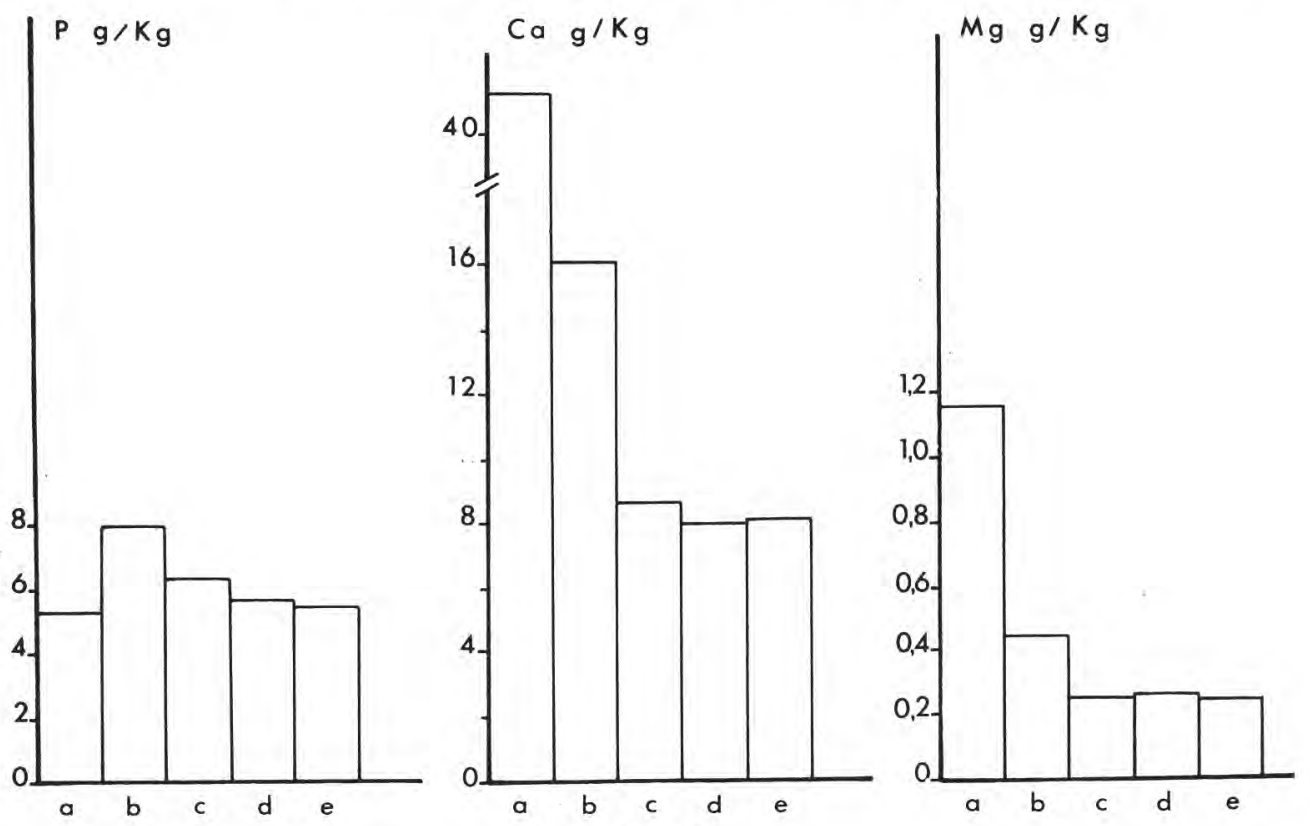

Fig. 4

Répartition du phosphore, du calcium et du magnésium dans un fromage affiné, Phosphorus, calcium and magnesium distribution in the ripened cheese.
$\mathrm{a}=$ Morge.
$\mathrm{b}=$ Croûte.
$\mathrm{d}=$ Zone intermédiaire.
$\mathrm{c}=$ Sous-croûte.
$\mathrm{e}=$ Centre.

de $\mathrm{pH}$; sa valeur est de 5,67 dans le centre, de 6,00 au niveau de la croûte et de 7,69 dans la morge.

Les teneurs en matière sèche de la croûte, de la partie sous-croûte, de la zone intermédiaire et du centre sont respectivement de 77,4\%,72,9\%, 65,7\% et $63,9 \%$ alors que celle de la morge est de $52,8 \%$.

La teneur en cendres, très importante dans la morge et la croûte, respectivement de $11,20 \%$ et $5,46 \%$, est assez homogène dans la pâte : $3,74 \%$ souscroûte et dans le centre et $3,88 \%$ dans la zone intermédiaire.

\section{Composition minérale du fromage}

a) Teneurs en phosphate, calcium et magnésium

La croûte présente une teneur en phosphore de $7,88 \mathrm{~g} / \mathrm{kg}$ et de $6,28 \mathrm{~g} / \mathrm{kg}$ pour la partie sous-croûte alors que celle de la morge est de $5,22 \mathrm{~g} / \mathrm{kg}$. Dans la zone intermédiaire et le centre, les différences sont peu sensibles : $5,57 \mathrm{~g}$ et $5,42 \mathrm{~g} / \mathrm{kg}$ (fig. 4).

La teneur en calcium de la croûte est pratiquement le double de celle de la partie sous-croûte, $16,10 \mathrm{~g} / \mathrm{kg}$ pour la première et 8,59 pour la seconde, alors que la valeur obtenue dans la morge est de $41,31 \mathrm{~g} / \mathrm{kg}$. Dans la zone intermé- 
diaire et le centre les teneurs sont respectivement de $7,93 \mathrm{~g} / \mathrm{kg}$ et de $8,07 \mathrm{~g} / \mathrm{kg}$. Le calcium migre donc de l'intérieur du fromage vers l'extérieur.

Le comportement du magnésium est identique à celui du calcium. Ainsi la quantité de magnésium contenue dans la morge est nettement supérieure à celle du reste du fromage. Les teneurs sont $1,17 \mathrm{~g} / \mathrm{kg}$ dans la morge, $0,44 \mathrm{~g} / \mathrm{kg}$ dans la croûte, $0,24 \mathrm{~g} / \mathrm{kg}$ dans la partie sous-croûte, $0,25 \mathrm{~g} / \mathrm{kg}$ dans la zone intermédiaire et $0,24 \mathrm{~g} / \mathrm{kg}$ dans le centre; les 3 dernières parties ont donc des teneurs identiques.

b) Teneur en sodium et potassium

La répartition du sodium est très différente des autres minéraux, en particulier au niveau de la croûte où les quantités sont inférieures à celles de l'intérieur du fromage (fig. 5). Les teneurs respectives dans la morge, la croûte, la partie sous-croûte, la zone intermédiaire et le centre sont de $9,85 \mathrm{~g}$ par $\mathrm{kg}, 3,73 \mathrm{~g} / \mathrm{kg}$, $4,30 \mathrm{~g} / \mathrm{kg}, 5,31 \mathrm{~g} / \mathrm{kg}$ et $4,73 \mathrm{~g} / \mathrm{kg}$. Il y a donc entre la croûte et la zone intermédiaire un gradient de concentration inverse des autres minéraux.

Le potassium a une répartition identique à celle du sodium. Ainsi sa teneur dans la croûte et la partie sous-croûte est inférieure à celle des autres parties.
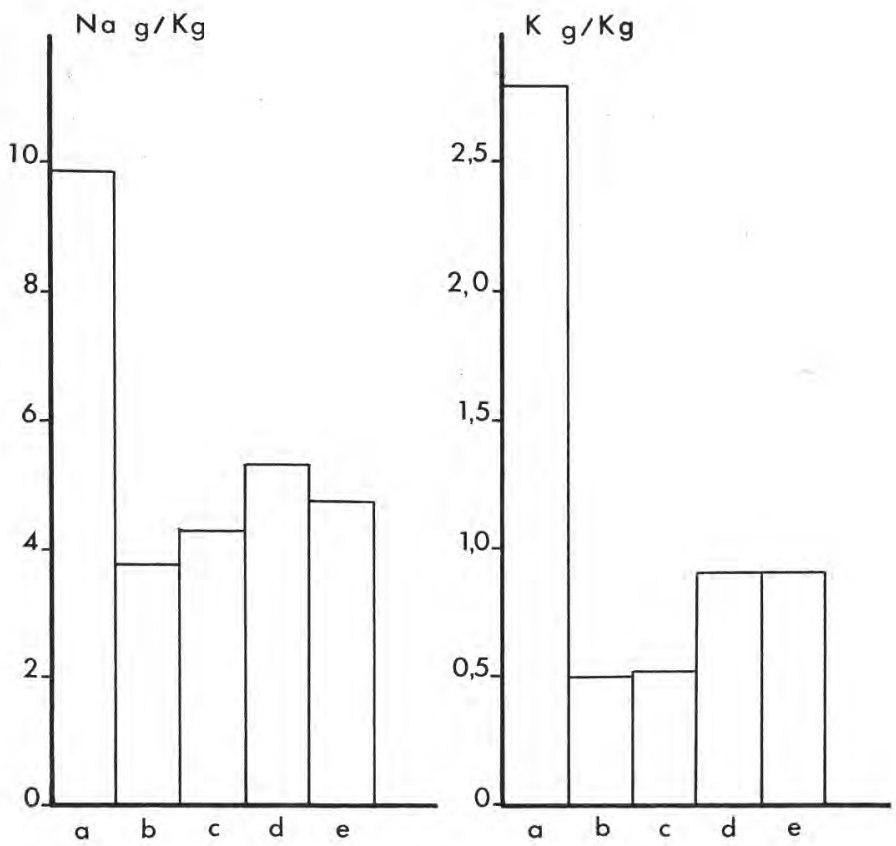

Fig. 5

Répartition du sodium et du potassium dans un fromage affiné.

Sodium and potassium distribution in the ripened cheese.
a $=$ Morge.
$\mathrm{b}=$ Croûte.
$\mathrm{d}=$ Zone intermédiaire.
$\mathrm{c}=$ Sous-croûte.
$\mathrm{e}=$ Centre. 
Les quantités de potassium sont de $0,50 \mathrm{~g} / \mathrm{kg}$ dans la croûte, $0,52 \mathrm{~g} / \mathrm{kg}$ dans la partie sous-croûte, $0,92 \mathrm{~g} / \mathrm{kg}$ dans la zone intermédiaire et la partie centrale soit 2 fois plus que dans la croûte mais près de 3 fois moins que dans la morge $(2,78 \mathrm{~g} / \mathrm{kg})$.

\section{c) Les oligo-éléments}

La croûte et la morge ont une teneur en zinc 4 à 6 fois supérieure à celle du reste du fromage (fig. 6). Les teneurs de la morge, de la croûte, partie souscroûte, zone intermédiaire et centre sont respectivement de 169,$00 ; 216,98 ; 73,93$; 47,31 et $45,46 \mathrm{mg} / \mathrm{kg}$.

Les teneurs en fer dans la morge, la croûte, la partie sous-croûte, la zone intermédiaire et le centre sont respectivement : 27,$36 ; 9,00 ; 3,25 ; 2,24$ et $2,02 \mathrm{mg} / \mathrm{kg}$. Le fer, comme les minéraux précédents, présente un gradient de concentration dans le fromage.

La teneur en cuivre de la morge est environ le double de celle du reste du fromage. Les valeurs obtenues sont de $16,25 \mathrm{mg} / \mathrm{kg}$ dans la morge, de $10,29 \mathrm{mg} / \mathrm{kg}$ dans la croûte, de $8,17 \mathrm{mg} / \mathrm{kg}$ dans la partie sous-croûte, de $7,87 \mathrm{mg} / \mathrm{kg}$ dans la zone intermédiaire et de $7,29 \mathrm{mg} / \mathrm{kg}$ dans le centre.

d) Proportion des minéraux dans chacune des parties du fromage

Le tableau 1 donne la composition moyenne du fromage, et la répartition du poids et des minéraux dans chacune des parties exprimées en pour cent (sauf pour
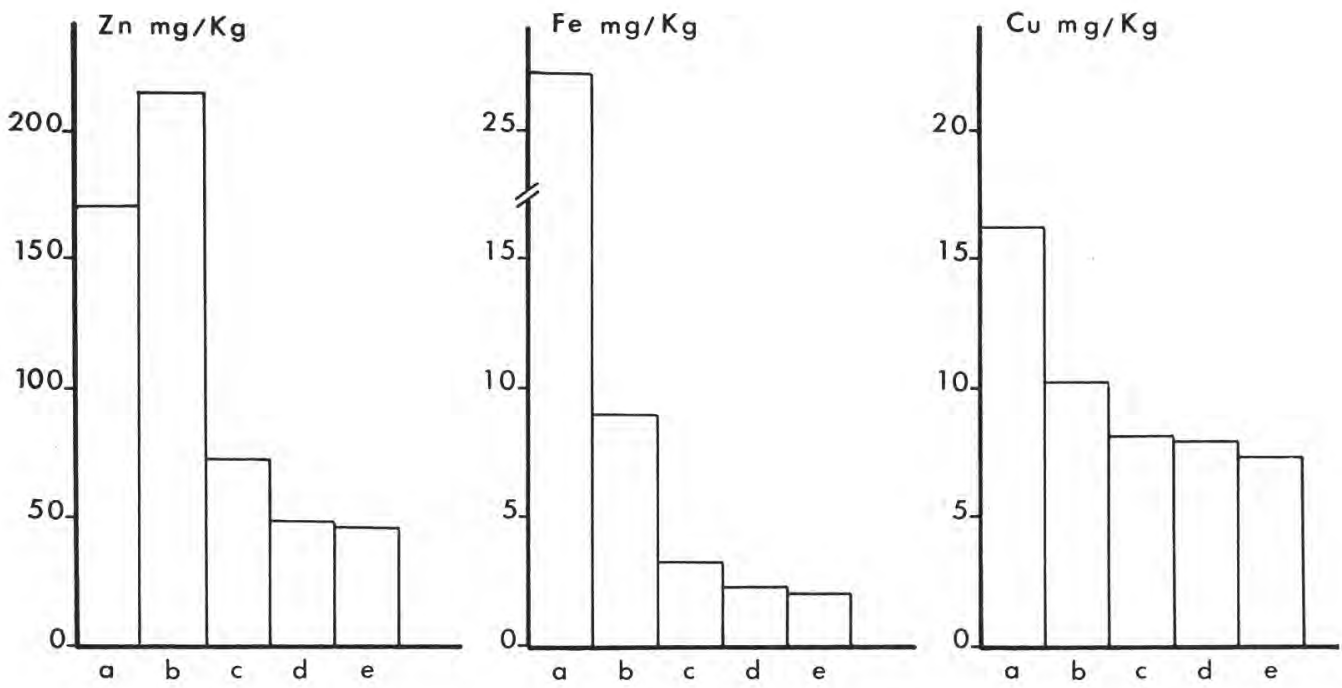

Fig. 6

Répartition du zinc, du fer et du cuivre dans un fromage affiné.

Zinc, iron and copper in the ripened cheese.
$\mathrm{a}=$ Morge.
$\mathrm{b}=$ Croûte .
$\mathrm{d}=$ Zone intermédiaire.
$\mathrm{c}=$ Sous-croûte.
$\mathrm{e}=$ Centre. 


\section{TABLEAU I}

Composition minérale moyenne du fromage

et répartition pondérale des minéraux dans les différentes parties du fromage Average Beaufort cheese composition in mineral substances

Distribution of phosphorus, calcium and other cations in various portions of a Beaufort cheese (as percent of total phosphorus, calcium, etc. present in the whole cheese)

\begin{tabular}{|c|c|c|c|c|c|c|c|c|c|}
\hline & Poids & $\mathrm{P}$ & $\mathrm{Ca}$ & $\mathrm{Mg}$ & $\mathrm{Na}$ & $\mathrm{K}$ & $\mathrm{Zn}$ & $\mathrm{Fe}$ & $\mathrm{Cu}$ \\
\hline \multirow{2}{*}{\multicolumn{2}{|c|}{ Composition moyenne }} & \multicolumn{5}{|c|}{$\mathrm{g} / \mathrm{kg}$} & \multicolumn{3}{|c|}{$\mathrm{mg} / \mathrm{kg}$} \\
\hline & & 5,99 & 8,72 & 0,26 & 4,79 & 0,82 & 63,4 & 2,8 & 7,8 \\
\hline & \multicolumn{9}{|c|}{ Répartition en \% } \\
\hline Croûte & 7,76 & 10,54 & 14,32 & 13,03 & 6,04 & 4,70 & 26,33 & 24,62 & 10,15 \\
\hline Sous-croûte & 15,95 & 17,25 & 15,70 & 15,00 & 14,32 & 10,15 & 18,43 & 18,30 & 16,54 \\
\hline Zone inter. & 35,89 & 34,44 & 32,60 & 34,48 & 39,79 & 40,15 & 26,54 & 28,34 & 35,87 \\
\hline Centre & 40,38 & 37,75 & 37,36 & 37,46 & 39,83 & 44,97 & 28,69 & 28,71 & 37,42 \\
\hline
\end{tabular}


la morge non représentative pondéralement compte tenu du mode de prélèvement). Ces résultats confirment la faible migration du phosphate et du cuivre dans la croûte qui contient respectivement $10,54 \%$ et $10,15 \%$ du total de ces minéraux pour $7,76 \%$ du poids. La croûte contient, par contre, $24,62 \%$ et $26,33 \%$ du fer et du zinc alors que la zone intermédiaire et le centre ont seulement des teneurs de $28,34 \%$ et $28,71 \%$ pour $35,80 \%$ et $40,38 \%$ du poids de fromage.

Le potassium présente une particularité puisque le centre contient $44,97 \%$ du total et la croûte seulement $4,70 \%$.

e) Composition minérale des saumures d'emmorgeage

Nous avons examiné l'évolution de la composition des saumures d'emmorgeage lors du traitement des fromages âgés soit de 8 jours, soit de 2 mois. L'emmorgeage des fromages de 8 jours est réalisé sur 150 meules de fromage à l'aide d'environ 8 litres de saumure usagée alors que celui de 2 mois a été effectué à l'aide d'une même quantité de saumure vierge et concerne 250 meules.

Comme le montre le tableau 2, la saumure utilisée pour l'emmorgeage des fromages de 8 jours présente une charge minérale initiale élevée ; elle s'enrichit toutefois en calcium et en moindre quantité en magnésium, phosphore, zinc, fer et potassium; les teneurs s'élèvent respectivement de 2160,0 à $3310,0 \mathrm{mg} / \mathrm{kg}$, de 53,4 à $63,8 \mathrm{mg} / \mathrm{kg}$, de 288,0 à $365,0 \mathrm{mg} / \mathrm{kg}$, de 6,9 à $10,1 \mathrm{mg} / \mathrm{kg}$, de 4,7 à $7,7 \mathrm{mg} / \mathrm{kg}$ et de 480,0 à $553,0 \mathrm{mg} / \mathrm{kg}$. Le cuivre migre relativement peu et 1'enrichissement de la saumure est très faible : 0,65 à $0,69 \mathrm{mg} / \mathrm{kg}$.

Les variations des teneurs en minéraux de la saumure vierge utilisée pour l'emmorgeage des fromages de 2 mois suivent la même évolution que celle observée à 8 jours, compte tenu de la différence dans le nombre de fromages traités. La teneur en calcium augmente de 124,0 à $2450,0 \mathrm{mg} / \mathrm{kg}$, celle du magnésium de 20,0 à $82,5 \mathrm{mg} / \mathrm{kg}$, du phosphore de 1 à $59 \mathrm{mg} / \mathrm{kg}$, du zinc de 0,3 à $7,2 \mathrm{mg} / \mathrm{kg}$, du fer de 0,2 à $5,0 \mathrm{mg} / \mathrm{kg}$ et du potassium de 31 à $274 \mathrm{mg} / \mathrm{kg}$.

\section{B. Migration du sodium, calcium et phosphate au cours de l'affinage}

\section{Matière sèche et cendres}

Dès 20 heures la matière sèche de la croûte est plus élevée que dans le reste du fromage : 60,11 \% dans le centre et 63,40\% dans la croûte (tabl. 3).

$\mathrm{Au}$ cours de l'affinage les teneurs en matière sèche de la partie sous-croûte évoluent de $60,9 \%$ à $62,6 \%$ et celle du centre de $60,3 \%$ à $61,2 \%$.

Dans la croûte l'évolution de la matière sèche est beaucoup plus importante : $63,4 \%$ à 20 heures et $73,4 \%$ à 8 semaines.

Les teneurs en cendres augmentent lentement dans le centre et la partie sous-croûte ; elles sont respectivement de 2,73 et $2,72 \mathrm{~g}$ p. 100 à 20 h et 3,04 et 3,21 mg p. 100 à 8 semaines. Durant la même période, les teneurs en cendres de la croûte augmentent de 2,84 et 4,75 g p. 100 ; cette augmentation des teneurs en cendres existe non seulement si l'on rapporte les cendres au poids frais, mais encore si on les rapporte à l'extrait sec du fromage bien que la différence soit plus faible du fait que ce dernier perd environ 7 à $8 \%$ de son poids frais au cours des 8 semaines. 


\section{TABLEAU II}

Evolution minérale $(\mathrm{mg} / \mathrm{kg})$ des saumures au cours des opérations d'emmorgeage

Evolution of minerals in the brine used for rubbing the cheese

\begin{tabular}{|c|c|c|c|c|c|c|c|c|}
\hline & $\mathbf{P}$ & $\mathrm{Ca}$ & $\mathrm{Mg}$ & $\mathrm{Na}$ & $\mathrm{K}$ & $\mathrm{Zn}$ & $\mathrm{Fe}$ & $\mathrm{Cu}$ \\
\hline Saumure avant les soins & 288 & 2160 & 53,4 & 77190 & 480 & 6,9 & 4,7 & 0,65 \\
\hline Saumure après les soins & 365 & 3310 & 63,8 & 77820 & 553 & 10,1 & 7,7 & 0,69 \\
\hline Saumure avant les soins & 1 & 124 & 20,0 & 96200 & 31 & 0,3 & 0,2 & 0,70 \\
\hline Saumure après les soins & 59 & 2450 & 82,5 & 97500 & 274 & 7,2 & 5,0 & 0,87 \\
\hline
\end{tabular}

(1) Saumure usagée avant et après traitement de 150 fromages de 8 jours.

(2) Saumure vierge avant et après traitement de 250 fromages de 2 mois. 


\section{TABLEAU III}

Evolution des teneurs en extrait sec et cendres (\%), sodium, calcium et phosphore $(\mathrm{g} / \mathrm{kg})$ du fromage au cours de l'affinage

Evolution of dry matter and ash ( $g$ per cent), and of sodium, calcium and phosphorus $(\mathrm{g} / \mathrm{kg})$ in cheese during ripening

\begin{tabular}{|c|c|c|c|c|c|c|c|}
\hline & & ES \% & Cendres \% & $\mathrm{Na} \mathrm{g} / \mathrm{kg}$ & $\mathrm{Ca} \mathrm{g/kg}$ & $\mathrm{P} \mathrm{g} / \mathrm{kg}$ & $\mathrm{Ca} / \mathrm{P}$ \\
\hline $20 \mathrm{~h}$ & $\begin{array}{l}1 \\
2 \\
3\end{array}$ & $\begin{array}{l}60,1 \\
59,7 \\
63,4\end{array}$ & $\begin{array}{l}2,73 \\
2,72 \\
2,84\end{array}$ & $\begin{array}{l}0,23 \\
0,24 \\
0,24\end{array}$ & $\begin{array}{l}8,55 \\
8,54 \\
9,07\end{array}$ & $\begin{array}{l}5,63 \\
5,65 \\
6,01\end{array}$ & $\begin{array}{l}1,48 \\
1,51 \\
1,50\end{array}$ \\
\hline $\begin{array}{l}\text { Après } \\
\text { saumure }\end{array}$ & $\begin{array}{l}1 \\
2 \\
3\end{array}$ & $\begin{array}{l}60,3 \\
60,9 \\
67,1\end{array}$ & $\begin{array}{l}2,78 \\
3,27 \\
3,43\end{array}$ & $\begin{array}{l}0,55 \\
2,78 \\
2,95\end{array}$ & $\begin{array}{l}8,48 \\
8,35 \\
9,04\end{array}$ & $\begin{array}{l}5,57 \\
5,59 \\
6,35\end{array}$ & $\begin{array}{l}1,52 \\
1,49 \\
1,42\end{array}$ \\
\hline $8 \mathrm{j}$. & $\begin{array}{l}1 \\
2 \\
3\end{array}$ & $\begin{array}{l}60,7 \\
61,2 \\
67,5\end{array}$ & $\begin{array}{l}2,84 \\
3,36 \\
3,90\end{array}$ & $\begin{array}{l}0,80 \\
3,25 \\
3,33\end{array}$ & $\begin{array}{r}8,73 \\
8,43 \\
10,00\end{array}$ & $\begin{array}{l}5,61 \\
5,53 \\
6,34\end{array}$ & $\begin{array}{l}1,55 \\
1,52 \\
1,57\end{array}$ \\
\hline $15 \mathrm{j}$. & $\begin{array}{l}1 \\
2 \\
3\end{array}$ & $\begin{array}{l}60,6 \\
61,2 \\
67,7\end{array}$ & $\begin{array}{l}2,88 \\
3,42 \\
3,99\end{array}$ & $\begin{array}{l}0,99 \\
3,58 \\
3,50\end{array}$ & $\begin{array}{r}8,64 \\
8,18 \\
10,09\end{array}$ & $\begin{array}{l}5,51 \\
5,52 \\
6,21\end{array}$ & $\begin{array}{l}1,56 \\
1,48 \\
1,62\end{array}$ \\
\hline 3 sem. & $\begin{array}{l}1 \\
2 \\
3\end{array}$ & $\begin{array}{l}60,2 \\
61,0 \\
70,0\end{array}$ & $\begin{array}{l}2,90 \\
3,55 \\
4,14\end{array}$ & $\begin{array}{l}1,04 \\
3,53 \\
3,26\end{array}$ & $\begin{array}{r}8,62 \\
7,88 \\
11,18\end{array}$ & $\begin{array}{l}5,53 \\
5,67 \\
6,56\end{array}$ & $\begin{array}{l}1,55 \\
1,37 \\
1,70\end{array}$ \\
\hline 4 sem. & $\begin{array}{l}1 \\
2 \\
3\end{array}$ & $\begin{array}{l}60,6 \\
61,1 \\
69,6\end{array}$ & $\begin{array}{l}2,93 \\
3,26 \\
4,27\end{array}$ & $\begin{array}{l}1,21 \\
3,27 \\
2,75\end{array}$ & $\begin{array}{r}8,76 \\
7,73 \\
12,32\end{array}$ & $\begin{array}{l}5,64 \\
5,54 \\
6,61\end{array}$ & $\begin{array}{l}1,55 \\
1,39 \\
1,86\end{array}$ \\
\hline 5 sem. & $\begin{array}{l}1 \\
2 \\
3\end{array}$ & $\begin{array}{l}61,1 \\
61,2 \\
71,4\end{array}$ & $\begin{array}{l}3,01 \\
3,22 \\
4,22\end{array}$ & $\begin{array}{l}1,50 \\
3,16 \\
2,82\end{array}$ & $\begin{array}{r}8,73 \\
7,99 \\
11,84\end{array}$ & $\begin{array}{l}5,43 \\
5,44 \\
6,63\end{array}$ & $\begin{array}{l}1,58 \\
1,46 \\
1,78\end{array}$ \\
\hline 6 sem. & $\begin{array}{l}1 \\
2 \\
3\end{array}$ & $\begin{array}{l}60,5 \\
61,3 \\
72,2\end{array}$ & $\begin{array}{l}2,90 \\
3,14 \\
4,51\end{array}$ & $\begin{array}{l}1,49 \\
2,84 \\
2,49\end{array}$ & $\begin{array}{r}8,63 \\
7,82 \\
13,60\end{array}$ & $\begin{array}{l}5,45 \\
5,35 \\
6,71\end{array}$ & $\begin{array}{l}1,58 \\
1,46 \\
2,02\end{array}$ \\
\hline 7 sem. & $\begin{array}{l}1 \\
2 \\
3\end{array}$ & $\begin{array}{l}61,0 \\
62,1 \\
74,5\end{array}$ & $\begin{array}{l}3,04 \\
3,14 \\
4,56\end{array}$ & $\begin{array}{l}1,64 \\
2,74 \\
2,25\end{array}$ & $\begin{array}{c}8,59 \\
7,94 \\
14,12\end{array}$ & $\begin{array}{l}5,59 \\
5,60 \\
7,33\end{array}$ & $\begin{array}{l}1,53 \\
1,41 \\
1,92\end{array}$ \\
\hline 8 sem. & $\begin{array}{l}1 \\
2 \\
3\end{array}$ & $\begin{array}{l}61,2 \\
62,6 \\
73,4\end{array}$ & $\begin{array}{l}3,06 \\
3,21 \\
4,75\end{array}$ & $\begin{array}{l}1,70 \\
3,17 \\
2,53\end{array}$ & $\begin{array}{r}8,62 \\
7,81 \\
14,67\end{array}$ & $\begin{array}{l}5,56 \\
5,49 \\
7,13\end{array}$ & $\begin{array}{l}1,55 \\
1,42 \\
2,05\end{array}$ \\
\hline
\end{tabular}

1 Centre.

2 Sous-croûte.

3 Croûte.

Cette étude a été réalisée à partir de sondes où la croûte représente 4 à $5 \%$ du poids, la partie sous-croûte 22 à $25 \%$ et le centre 70 à $74 \%$ (fig. 2). 


\section{Migration du sodium, calcium et phosphate au cours de l'affinage}

Après saumurage les quantités de sodium augmentent mais en présentant un gradient de concentration de l'intérieur vers l'extérieur. A partir de 15 jours, la teneur de la croûte devient inférieure à celle de la partie sous-croûte; ce phénomène s'accentue au cours de l'affinage. Ainsi à 8 semaines les teneurs en sodium sont de $1,70 \mathrm{~g} / \mathrm{kg}$ dans le centre, $3,17 \mathrm{~g} / \mathrm{kg}$ dans la partie sous-croûte et $2,53 \mathrm{~g} / \mathrm{kg}$ dans la croûte.

Après saumurage, les teneurs en calcium de la croûte et de la partie souscroûte sont de 9,04 et $8,35 \mathrm{~g} / \mathrm{kg}$. Les valeurs obtenues en rapportant la quantité de calcium du centre à l'extrait sec (corrigé des variations de la teneur en cendres) de la croûte et partie sous-croûte permettent d'évaluer respectivement les pertes de calcium dans la saumure à $0,30 \mathrm{~g} / \mathrm{kg}$ et $0,15 \mathrm{~g} / \mathrm{kg}$, soit $0,5-0,6 \%$ du calcium total du fromage.

A partir de 15 jours, la croûte s'enrichit en calcium, alors que la partie souscroûte s'appauvrit; les teneurs à 15 jours sont de $8,64 \mathrm{~g} / \mathrm{kg}$ dans le centre, $8,18 \mathrm{~g} / \mathrm{kg}$ dans la partie sous-croûte et $10,09 \mathrm{~g} / \mathrm{kg}$ dans la croûte. Au cours de l'affinage le phénomène d'accumulation dans la croûte va s'accentuer alors que la partie sous-croûte va s'appauvrir constamment. A 8 semaines les teneurs sont de $8,62 \mathrm{~g} / \mathrm{kg}$ dans le centre, $7,81 \mathrm{~g} / \mathrm{kg}$ dans la partie sous-croûte et $14,67 \mathrm{~g} / \mathrm{kg}$ dans la croûte.

Les teneurs en phosphore sont beaucoup plus homogènes que celles du calcium. A partir de 3 semaines, la teneur de la croûte augmente dans de faibles proportions; elle passe de $6,56 \mathrm{~g} / \mathrm{kg}$ à $7,13 \mathrm{~g} / \mathrm{kg}$ à 8 semaines. La partie souscroûte et le centre ont des teneurs pratiquement constantes aux environs de $5,50 \mathrm{~g} / \mathrm{kg}$.

Le rapport $\mathrm{Ca} / \mathrm{P}$ est de 1,50 environ dans le fromage à $20 \mathrm{~h}$ et reste constant dans le centre au cours des 2 mois d'affinage. A partir de 15 jours ce rapport diminue dans la partie sous-croûte et atteint 1,42 alors que dans la croûte il augmente considérablement et se situe à 2,05 à 8 semaines.

\section{Discussion}

L'étude de la répartition des minéraux dans le fromage met en évidence des migrations dont le sens et l'amplitude diffèrent selon l'élément envisagé. Le transfert de calcium dans la morge est significatif $(41,31 \mathrm{~g} / \mathrm{kg})$ alors que celui du phosphore est très faible. Dans les fromages à pâte molle de type Camembert, le déplacement du calcium va de pair avec celui du phosphore pour donner un phosphate tricalcique (LE GRAET et al., 1983). Dans le cas du fromage de Beaufort il ne peut s'agir de phosphate tricalcique compte tenu du rapport $\mathrm{Ca} / \mathrm{P}$ voisin de 9,4 obtenu dans la morge. La recherche de $\mathrm{CO}_{2}$ par solubilisation acide des minéraux de la croûte et surtout de la morge a montré que ce gaz était présent en quantité importante. $\mathrm{Le} \mathrm{CO}_{2}$ proviendrait essentiellement de la flore établie dans la morge comme l'ont montré Blanc et al. (1982). Une partie de ce $\mathrm{CO}_{2}$ pourrait donc se combiner au calcium et au magnésium pour former, compte tenu du $\mathrm{pH}$ élevé, un carbonate insoluble. La précipitation du carbonate aurait 
pour conséquence une diminution de la teneur en calcium et magnésium solubles, ce qui favoriserait leur transfert à partir des zones plus internes.

La faible augmentation des teneurs en phosphore dans la croûte pourrait correspondre à de petites quantités de phosphate tricalcique ou à un phosphate associé à d'autres cations. Le calcium et le magnésium se combinent préférentiellement au $\mathrm{CO}_{2}$, ce qui se traduit par un transfert très limité de l'ion phosphate.

Le fer et le zinc migrent dans des proportions plus importantes que le calcium et le magnésium comme le montre l'analyse des teneurs de la croûte et de la morge 5 fois supérieures à celles du centre. Néanmoins, en ce qui concerne le fer, des contaminations sont toujours possibles (au niveau du matériel, des soins), ce qui expliquerait une proportion plus importante de cet élément dans la morge, comme le montre le rapport $\frac{\text { teneur en fer de la croûte }}{\text { teneur en fer du centre }}=\frac{27,3}{2,02}$ bien plus élevé que celui des autres minéraux. Le cuivre est par contre beaucoup moins mobile car plus fortement fixé aux protéines et peptides. L'origine de l'importante teneur minérale de la morge ne peut être attribuée aux saumures comme le montre le tableau 1 puisque ces dernières s'enrichissent en minéraux au cours des soins apportés aux fromages. Les emmorgeages successifs réalisés à l'aide d'une saumure à $\mathrm{pH}$ basique et l'atmosphère d'affinage fortement ammoniacale créent les conditions favorables à la précipitation du fer, du zinc et du cuivre, à la surface du fromage sous forme d'hydroxydes, de phosphates et carbonates insolubles en l'absence de chélatant tel que le citrate. La formation de ces sels insolubles entraîne une réduction de la teneur en minéraux de la phase soluble contenue dans la morge ; mais l'équilibre se rétablit par suite de la migration des minéraux des zones internes vers la surface.

La forte teneur de la morge en sodium peut avoir deux origines, les opérations successives d'emmorgeage qui apportent du chlorure de sodium, et la faible teneur en matière sèche qui implique une quantité de phase soluble c'est-à-dire d'eau solvante plus importante que dans le reste du fromage. Ceci permet de solubiliser davantage de chlorure de sodium. Cette interprétation doit malgré tout tenir compte de la composition de la morge qui est très différente de celle du reste du fromage et fait donc intervenir d'autres facteurs, tels que la teneur en matière grasse beaucoup plus faible et l'eau de constitution de la flore bactérienne non disponible ; par ailleurs la méthode de dosage de l'extrait sec ne permet pas d'apprécier les pertes en substances volatiles. Ces facteurs sont donc susceptibles de modifier sensiblement les rapports eau solvante-eau liée et par conséquent la quantité de chlorure de sodium solubilisé.

Le gradient de concentration en sodium de la croûte, sous-croûte et zone intermédiaire pourrait aussi s'expliquer par des différences en eau solvante contenue dans ces diverses parties. Ce phénomène a déjà été observé par HuREL et MoCQUOT (1947) sur des fromages à pâte molle et appelé « inversion des salures 》.

L'accumulation du potassium dans la morge est liée d'une part à la phase solvante beaucoup plus importante que dans le reste du fromage et, d'autre part, à la flore de surface dont le développement nécessite, entre autre, la présence de sodium et de potassium.

L'emmorgeage se traduit par un enrichissement minéral de la saumure aux dépens du fromage; ces résultats confirment ceux de LACRAMPE et al. (1971) et TERRE et al. (1985). Ces transferts sont fonction de la charge minérale initiale 
de la saumure utilisée (TERRE et al., 1985), de la teneur de la morge du fromage et du nombre de fromages traités. Néanmoins, dans le cas du Beaufort, le bilan des transferts minéraux, en ce qui concerne les saumures d'emmorgeage, demeure quantitativement faible si l'on admet que 8 litres de saumure permettent d'emmorger 150 à 250 fromages d'environ $45 \mathrm{~kg}$ chacun; ces opérations sont renouvelées à raison d'une à deux fois par semaine durant 7 à 8 mois.

L'ensemble de ces résultats met en évidence une grande hétérogénéité physico-chimique $(\mathrm{pH}$, teneur en eau, composition minérale) dans le fromage notamment dans la partie sous-croûte et la morge. Ces modifications physico-chimiques induites par les traitements de surface et le caractère alcalin de l'atmosphère sont peut-être déterminantes pour le déroulement de l'affinage tant au niveau de la flore que de l'activité enzymatique et des caractéristiques rhéologiques de la pâte.

L'influence de l'emmorgeage sur l'humidité de surface et sur la flore ainsi que ses conséquences sur le système enzymatique mériterait donc une étude plus approfondie.

Reçu le 27 novembre 1985.

Accepté pour publication le 15 juin 1986.

\section{Références bibliographiques}

Blanc B., Bosset J.O., Martin B., Jimeno J., 1982. Echanges gazeux à la surface du fromage de Gruyère en cours de maturation. Schweiz Milch. Forsch., 12 (30-34), 3-7.

Gueguen L., 1979. Apports minéraux par le lait et les produits laitiers. Cah. Nutr. Diét., 14 (3), 213-217.

Guittonneau G., Chevalter R., 1934. Sur les équilibres calcophosphoriques réalisés dans les fromages. C.R. Acad. Sci., 199, 801-803.

Hurel C., Mocouot G., 1947. Etude comparée des fromages de Livarot et de Pont-1'Evêque. Lait, 27 (263-264), 131-141.

Koestler G., 1943. Ueber Teigverschiedenheiten in ein und demselben Emmentalerkase. Ann. Agric. Suisse, 44 (3), 266-289. (Dairy Sci. Abst., 5, 151).

Lacrampe J.L., Hardy J., Ramet J.P., Weber F., 1971. Contribution à l'étude de l'évolution chimique et du traitement des saumures de fromageries, Lait, 51 (503-504), 158-175.

Le Graet Y., Lepienne A., Brulé G., Ducruet P., 1983. Migration du calcium et des phosphates inorganiques dans les fromages à pâte molle de type Camembert au cours de l'affinage. Lait, 63 (629-630), 317-332.

MatTSON S., 1954. Kalcium-och Fosforhalten : hardost. Svenska Mejeritidn, 46 (2), 15-19. (Dairy Sci. Abst., 16, 590).

Metche M., Fanni J., 1978. Rôle de la flore fongique dans l'accumulation du calcium et du phosphore à la surface des fromages du type Camembert. Lait, 58 (77), 336-354.

Resmini P., Volonterio G., AnNibaloi S., Ferri G., 1974. Study of salt diffusion in Parmigiano-Reggiano cheese using $\mathrm{Na}^{36} \mathrm{Cl}$. Sci. Tec. Latt. Casearia, 25 (4), 149-166. (Dairy Sci. Abst., 37, 632).

Terre E., Le Graet Y., Brulé G., Maubois J.L., 1985. Etude du transfert des solutés des fromages à pâte molle dans les saumures. Intérêt du traitement par ultrafiltration sur membrane. Tech. Lait., (997), 39-47. 